\title{
Substrate-Dependent Differences in the Crystal Structures and Optical Properties of ZnSe Nanowires
}

\author{
Keumyoung Seo, ${ }^{1}$ Jihye Bong, ${ }^{1}$ Jae-Woo Kim, ${ }^{2,3}$ Yoon-Ho Song, ${ }^{2,3}$ \\ Yong Ho Shin, ${ }^{4}$ Yongmin Kim, ${ }^{4}$ and Sanghyun $\mathrm{Ju}^{1}$ \\ ${ }^{1}$ Department of Physics, Kyonggi University, Suwon, Gyeonggi-do 443-760, Republic of Korea \\ ${ }^{2}$ School of Advanced Device Engineering, University of Science \& Technology, 217 Gajeong-ro, Yuseong-gu, \\ Daejeon 305-350, Republic of Korea \\ ${ }^{3}$ Nano Electron-Source Creative Research Center, Electronics and Telecommunications Research Institute, 218 Gajeong-ro, \\ Yuseong-gu, Daejeon 305-700, Republic of Korea \\ ${ }^{4}$ Department of Applied Physics and Institute of Nanoscience and Biotechnology, Dankook University, \\ Yongin 448-701, Republic of Korea
}

Correspondence should be addressed to Sanghyun Ju; shju@kgu.ac.kr

Received 20 August 2015; Accepted 21 September 2015

Academic Editor: Magnus Willander

Copyright (C) 2015 Keumyoung Seo et al. This is an open access article distributed under the Creative Commons Attribution License, which permits unrestricted use, distribution, and reproduction in any medium, provided the original work is properly cited.

\begin{abstract}
The optical and structural properties of $\mathrm{ZnSe}$ nanowires directly grown on three different substrates, $\mathrm{SiO}_{2}$, ITO, and graphene, were investigated. $\mathrm{ZnSe}$ nanowires grown on graphene and $\mathrm{SiO}_{2}$ were found to have cubic structures, while $\mathrm{ZnSe}$ nanowires grown on ITO had a mixed cubic and hexagonal structure. The main peaks in the photoluminescence spectra of ZnSe nanowires grown on $\mathrm{SiO}_{2}$, ITO, and graphene were located at 459,627 , and $627 / 460 \mathrm{~nm}$, respectively. In addition, a field-emission light-emitting device was fabricated using $\mathrm{ZnSe}$ nanowires as a phosphor and graphene as an electrode. The device showed a red emission peak with Commission Internationale de L'Eclairage coordinates of $(0.621,0.315)$.
\end{abstract}

\section{Introduction}

One-dimensional single-crystal nanowires have large aspect ratios (length-to-width ratio) and exhibit a variety of interesting electrical and optical properties not observed in conventional bulk and thin film materials. These properties have led to the recent application of nanowires in conceptually new transistors, displays, lasers, photodetectors, and photovoltaic devices [1-3]. In particular, recent efforts to develop flexible and/or transparent displays have included research into developing light-emitting diodes (LEDs), field-emitting displays (FEDs), and electroluminescence (EL) devices with nanowires [4-6]. Indium tin oxide (ITO) and indium zinc oxide (IZO) are the materials currently being used for transparent electrodes, which are essential to the development of transparent displays. However, ITO and IZO materials have several limitations: they are available in limited quantities and their poor flexibilities lead to cracking, which deteriorates and reduces device reliabilities. To overcome these limitations, several studies have investigated transparent electrodes made from graphene thin films [7-9]. Graphene is a two-dimensional carbon nanomaterial. Recently, it has attracted increased attention as a possible electrode material for use in organic light-emitting diodes (OLEDs), solar cells, touch screens, and nanogenerators because of its outstanding properties, such as high electron mobility, transparency, conductivity, and flexibility [10-12].

ZnSe nanowires are excellent candidates for novel optoelectronic and electronic devices because they have a wide direct band gap of $2.7 \mathrm{eV}$ at $300 \mathrm{~K}$ and large surface-tovolume ratios. $\mathrm{ZnSe}$ nanowires are particularly suitable for use in applications such as diode lasers, light-emitting diodes, and photodetectors [13-15]. Because of their numerous applications, the structural and optical properties of $\mathrm{ZnSe}$ nanowires grown on $\mathrm{Si}, \mathrm{Al}_{2} \mathrm{O}_{3}$, and $\mathrm{SiO}_{2}$ substrates have been previously investigated. Several studies have explored the growth of $\operatorname{In}_{x} \mathrm{Ga}_{1-x} \mathrm{As}, \mathrm{SiC}$, and $\mathrm{In}_{2} \mathrm{O}_{3}$ nanowires on 
graphene [16-18] but none has studied the direct growth of $\mathrm{ZnSe}$ nanowires on graphene. Thus, observations of the crystal and optical properties of $\mathrm{ZnSe}$ nanowires grown on graphene thin films are of particular interest. The advantage of growing nanowires directly on graphene is that it facilitates the formation of Ohmic contacts between the nanowires and graphene $[17,18]$. In addition, hybrid graphene-ZnSe nanowire structures could be used in flexible and/or transparent displays.

This study presents a comparative analysis of ZnSe nanowires grown directly on graphene and those grown on $\mathrm{SiO}_{2}$ and ITO. The crystal and optical characteristics of $\mathrm{ZnSe}$ nanowires grown on graphene were examined using photoluminescence (PL), X-ray diffraction (XRD), and highresolution transmission electron microscopy (HR-TEM). In addition, a field-emission light-emitting device was fabricated using a ZnSe nanowire phosphor and transparent graphene electrodes to verify the luminescence properties of the $\mathrm{ZnSe}$ nanowires.

\section{Materials and Methods}

ZnSe nanowires were grown on three different substrates, $\mathrm{SiO}_{2}$, ITO, and graphene, by chemical vapor deposition (CVD). $\mathrm{SiO}_{2}$ was thermally grown to a thickness of $300 \mathrm{~nm}$ on a Si wafer. An ITO thin film (with a thickness of $\sim 100 \mathrm{~nm}$ ) was deposited on a quartz wafer using the radio frequency (RF) magnetron sputtering method. Graphene was grown on copper $(\mathrm{Cu})$ foil (Sigma-Aldrich, $25 \mu \mathrm{m}$ thick) and then transferred to a quartz wafer. Graphene was grown by placing the $\mathrm{Cu}$ foil in a CVD chamber with $100 \mathrm{sccm}$ of $\mathrm{H}_{2}$ gas and held at $1020^{\circ} \mathrm{C}$ for $90 \mathrm{~min} .20 \mathrm{sccm}$ of $\mathrm{CH}_{4}$ gas was then passed through the chamber for another $30 \mathrm{~min}$. The temperature of the CVD chamber was reduced at a rate of $150^{\circ} \mathrm{C} / \mathrm{min}$. Polymethyl methacrylate (PMMA) was then coated onto the graphene grown on the $\mathrm{Cu}$ foil and the $\mathrm{Cu}$ was removed using a wet-etching process. Subsequently, the graphene/PMMA was transferred to a quartz substrate and $20 \mathrm{sccm}$ of Ar gas was passed through the vacuum $\left(7.7 \times 10^{-2}\right.$ Torr $)$ at $400^{\circ} \mathrm{C}$ for $20 \mathrm{~min}$ to remove the PMMA. By repeating the transfer process five times, a five-layer graphene thin film on a quartz substrate was obtained. Au seed dispersion was achieved by placing a drop of Au seed solution, diluted to a volume ratio of 15:1 (IPA: Au colloid), on each substrate, namely, $\mathrm{SiO}_{2}$, ITO, and graphene. Au-coated $\mathrm{SiO}_{2}$, ITO, and graphene substrates, together with ZnSe (Sigma Aldrich, 99.995\%) powder, were placed in zones 1 and 2 of the CVD chamber. The temperatures of zones 1 and 2 were increased to $620^{\circ} \mathrm{C}$ and $920^{\circ} \mathrm{C}$, respectively, and $90 \mathrm{sccm}$ of Ar gas was passed through the chamber for $30 \mathrm{~min}$ to grow the ZnSe nanowires.

Low-temperature PL measurements were taken over a temperature range of $6-300 \mathrm{~K}$ by mounting samples on the cold-finger of a closed-cycle refrigerator. A $50 \mathrm{~cm}$ spectrometer equipped with a liquid nitrogen cooled charge coupled device $(2048 \times 512$ pixels $)$ was used to record PL spectra. PL emissions were observed across the entire spectral range using a 150 line $/ \mathrm{mm}$ (resolution $\sim 1.3 \mathrm{meV}$ ) ruled grating and in the band-edge region using a 1200 line/mm (resolution $\sim 0.1 \mathrm{meV}$ ) ruled grating. A $325 \mathrm{~nm} \mathrm{He}-\mathrm{Cd}$ laser was used to excite the ZnSe nanowires.

Figure 1 shows field-emission scanning electron microscope (FE-SEM) images of $\mathrm{ZnSe}$ nanowires grown on $\mathrm{SiO}_{2}$, ITO, and 5-layered graphene, as well as optical images of the light-emitting states of the $\mathrm{ZnSe}$ nanowires when illuminated by ultraviolet (UV) light $(365 \mathrm{~nm}, 8 \mathrm{~W})$. The $\mathrm{ZnSe}$ nanowires were successfully grown on the three different substrates, showing no signs of flakes or particles. The average diameters of the ZnSe nanowires were between 20 and $60 \mathrm{~nm}$ and the average lengths were greater than $5 \mu \mathrm{m}$. As shown in the optical images of the light emissions of the ZnSe nanowires grown on $1 \times 1$ inch substrates, under UV illumination $\mathrm{ZnSe}$ nanowires on thin film $\mathrm{SiO}_{2}$ were not luminescent, whereas ZnSe nanowires on thin film ITO exhibited a reddish-orange luminescence. The $\mathrm{ZnSe}$ nanowires on graphene also showed a reddish-orange luminescence, which was restricted to the area of graphene covered in $\mathrm{ZnSe}$ nanowires.

To identify the crystallographic differences between the $\mathrm{ZnSe}$ nanowires grown on thin films of $\mathrm{SiO}_{2}$, ITO, and graphene, XRD analysis was performed (Figure 2(a)). Figure 2(a) shows that the $\mathrm{ZnSe}$ nanowires on both $\mathrm{SiO}_{2}$ and graphene had cubic structures $(a=5.66882)$. The main peaks of those nanowires were (111), (220), (311), (400), and (331), located at $27.2^{\circ}, 45.2^{\circ}, 53.6^{\circ}, 65.8^{\circ}$, and $72.6^{\circ}$, respectively (JCPDS 37-1463). On the other hand, the ZnSe nanowires grown on ITO demonstrated peaks from both cubic ( $a=$ 5.66882) and hexagonal ( $a=3.974, c=6.506)$ structures. In addition to the peaks of the cubic-structured $\mathrm{ZnSe}$ nanowires seen in samples grown on $\mathrm{SiO}_{2}$ and graphene, ZnSe nanowires grown in ITO demonstrated the (100), (101), (102), (103), (203), and (105) peaks of hexagonal-structured ZnSe nanowires at $25.6^{\circ}, 29.0^{\circ}, 37.7^{\circ}, 49.2^{\circ}, 69.1^{\circ}$, and $77.7^{\circ}$, respectively (JCPDS 80-0008). The (222), (400), (440), and (622) peaks observed at $30.8^{\circ}, 35.7^{\circ}, 51.4^{\circ}$, and $61.1^{\circ}$, respectively (not shown in this figure), match the peaks of the ITO substrate (JCPDS 6-0416).

Figure 2(b) shows HR-TEM images of $\mathrm{ZnSe}$ nanowires grown on thin films of $\mathrm{SiO}_{2}$, ITO, and graphene. Similar to the $\mathrm{XRD}$ results, these images show that the $\mathrm{ZnSe}$ nanowires grown on thin film $\mathrm{SiO}_{2}$ had a cubic structure and a growth direction of [111]. In contrast, the $\mathrm{ZnSe}$ nanowires grown on thin film ITO had both hexagonal structure characteristics, demonstrating a growth direction of [100], and cubic structure characteristics, demonstrating a growth direction of [200]. In other words, the $\mathrm{ZnSe}$ nanowires grown on ITO exhibited two different crystalline structures and growth directions, whereas the $\mathrm{ZnSe}$ nanowires grown on $\mathrm{SiO}_{2}$ showed a single crystalline structure and growth direction. Although the ZnSe nanowires grown on graphene had a primarily cubic structure and [111] growth direction (similar to $\mathrm{ZnSe}$ nanowires on $\mathrm{SiO}_{2}$ ), some areas of the sample showed a twinned structure.

Figure 3 presents low-temperature PL spectra measured at $6 \mathrm{~K}$. As shown in Figure 3(a), at the band edge, blue luminescence (centered at $2.70 \mathrm{eV}(459 \mathrm{~nm})$ ) dominated the red luminescence (centered at $1.98 \mathrm{eV}(625 \mathrm{~nm})$ ) of the $\mathrm{ZnSe}$ nanowires grown on $\mathrm{SiO}_{2}$. The most intense peak of the $\mathrm{ZnSe}$ 


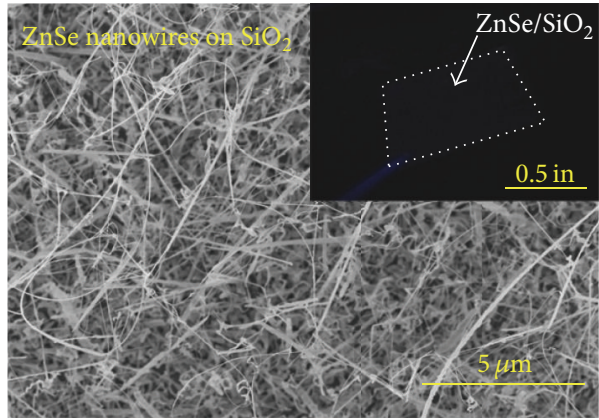

(a)

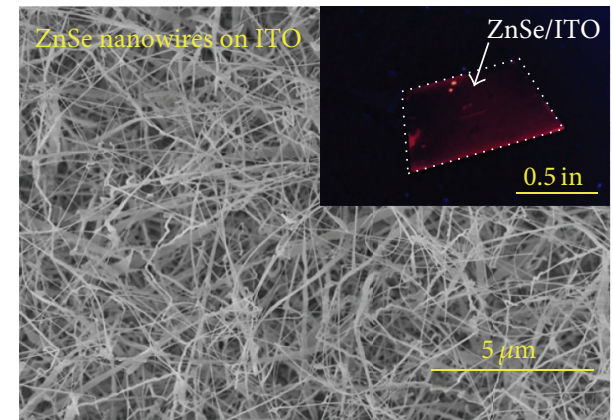

(b)

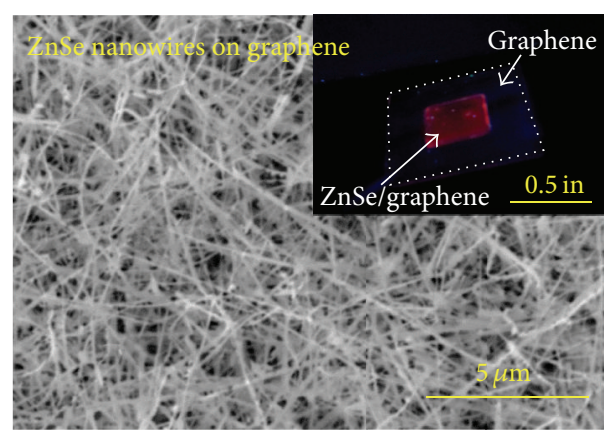

(c)

Figure 1: FE-SEM images of ZnSe nanowires grown on (a) $\mathrm{SiO}_{2}$, (b) ITO, and (c) graphene. The insets show optical luminescence images of samples under UV illumination.

nanowires fabricated on $\mathrm{SiO}_{2}$ was at $2.6981 \mathrm{eV}$ and is associated with a donor-acceptor pair (DAP) transition. Lowerenergy shoulder peaks, observed at 2.6668, 2.6355, and $2.6042 \mathrm{eV}$, correspond to longitudinal optical (LO) phonon replicas of donor-acceptor pairs (DAPs) and were spaced $31.3 \mathrm{meV}$ apart. Figure 3(b) shows an expanded view of the higher energy side-peaks, wherein the $\mathrm{I}_{1}$ and $\mathrm{I}_{2}$ peaks, as well as the free exciton (broken arrow) peak that was observed as a small bump in the spectrum at $2.8014 \mathrm{eV}$, are resolved $[19,20]$. It is known that $I_{2}$ lines, which are associated with excitons bound to neutral donors, can be found only in crystal of the highest quality [21]. An exciton bound to a neutral acceptor is responsible for the $\mathrm{I}_{1}$ transition as well as its LO phonon replica $\left(\mathrm{I}_{1}-\mathrm{LO}\right)$ at $2.7525 \mathrm{eV}$. Assignments of these sharp band-edge features were based on characteristics of a uniform and defect-free zinc-blende cubic structure [22, 23]. These assumptions were confirmed by the XRD and HRTEM results shown in Figure 2. In the case of the $\mathrm{ZnSe}$ nanowires grown on ITO, the main peak was located at $1.977 \mathrm{eV}(627 \mathrm{~nm})$ and lacked any band-edge transitions. The broad spectral feature centered at $627 \mathrm{~nm}$ is associated with a self-activated emission due to donors and deep acceptor pair transitions resulting from $\mathrm{Zn}$-vacancies or interstitial states $[15,24]$. In our case, such vacancies or interstitial states may be the result of crystal interfaces between hexagonal and cubic lattices, as shown in Figure 2(b). Such mixed phases deteriorate crystal quality and prevent PL emissions from having sharp band-edge structures.

On the other hand, the main transition peaks of the ZnSe nanowires grown on graphene had two distinct regions, namely, a band-edge related peak at $2.695 \mathrm{eV}(460 \mathrm{~nm})$ and a broad feature at $1.977 \mathrm{eV}(627 \mathrm{~nm})$. Unlike $\mathrm{ZnSe}$ nanowires grown on ITO, ZnSe nanowires grown on graphene had a single-phase cubic zinc-blende structure. However, it also demonstrated a twinned structure, as shown in Figure 2(b). Comparable to the $\mathrm{ZnSe}$ nanowires grown on graphene here, twinned structure $\mathrm{ZnSe}$ nanowires grown on alumina and $\mathrm{Si}$ substrates have been shown to have strong red emissions and relatively weak band-edge emissions $[25,26]$.

In addition, we verified that $\mathrm{ZnSe}$ nanowires grown on graphene can be used in light-emitting devices. Analyzing the optical emission processes of $\mathrm{ZnSe}$ nanowires should facilitate the development of $\mathrm{ZnSe}$ nanowire-based lightemitting devices. Figure 4(a) presents field-emission current densities as a function of the electric field applied to the device. As illustrated in Figure 4(a), electricity started to flow when the electric field was $1.5 \mathrm{~V} / \mu \mathrm{m}$ and the field-emission current density increased exponentially as the electric field was increased to $2.2 \mathrm{~V} / \mu \mathrm{m}$. When the applied field was equal to $2.2 \mathrm{~V} / \mu \mathrm{m}$, the emission current density was $\sim 30 \mu \mathrm{A} / \mathrm{cm}^{2}$. The field-emission- (FE-) current-voltage characteristics were obtained using the Fowler-Nordheim equation, $J=$ $\left(A \beta^{2} E^{2} / \Phi\right) \exp \left[-B \Phi^{3 / 2}(\beta E)^{-1}\right]$, where $J$ is current density, $E$ is applied electric field, $A$ is $1.56 \times 10^{-10}\left(\mathrm{AV}^{-2} \mathrm{eV}\right), B$ is $6.83 \times$ $10^{3}\left(\mathrm{VeV}^{-3 / 2} \mu \mathrm{m}^{-1}\right), \Phi$ is the work function of the $\mathrm{ZnSe}$ emitting material $(4.84 \mathrm{eV})$ [27], and $\beta$ is the field-enhancement factor, which is related to emitter geometry, crustal structure, and spatial distribution of the emitting centers [28]. Note that $\beta$ was extracted from the slope of a $\ln \left(J / E^{2}\right)$ versus $(1 / E)$ 


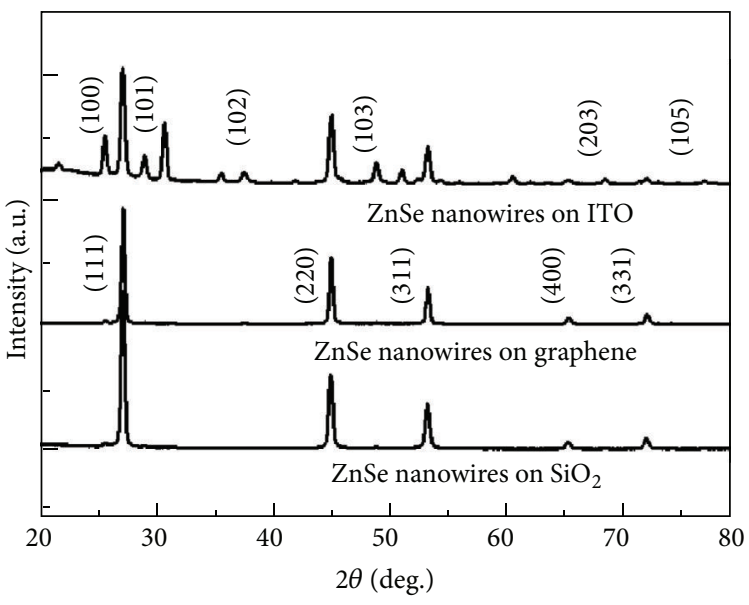

(a)
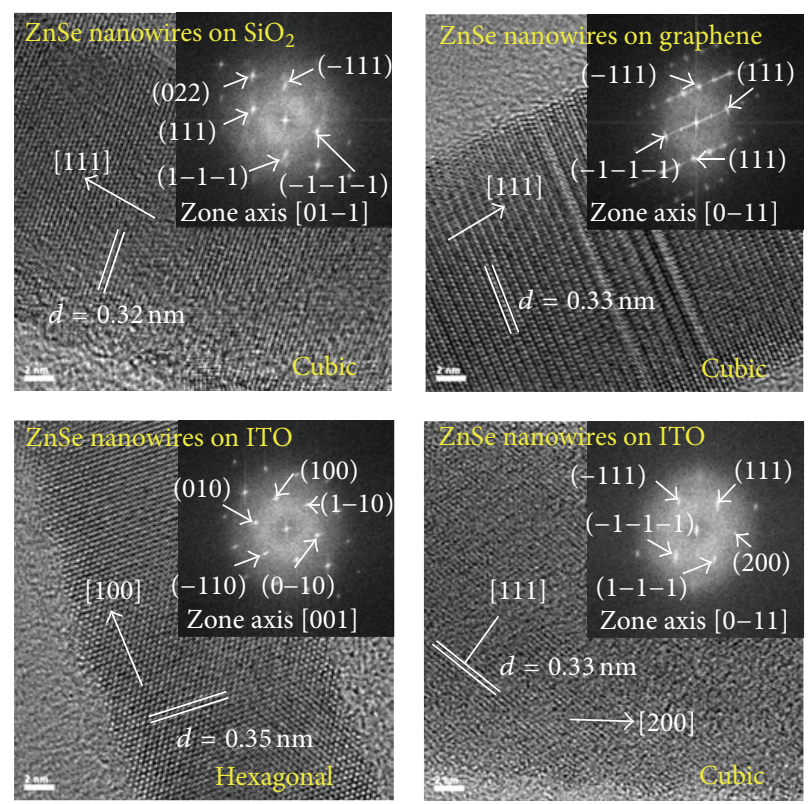

(b)

Figure 2: (a) XRD patterns of ZnSe nanowires grown on $\mathrm{SiO}_{2}$, ITO, and graphene. (b) Representative HR-TEM images of ZnSe nanowires grown on $\mathrm{SiO}_{2}$, ITO, and graphene.

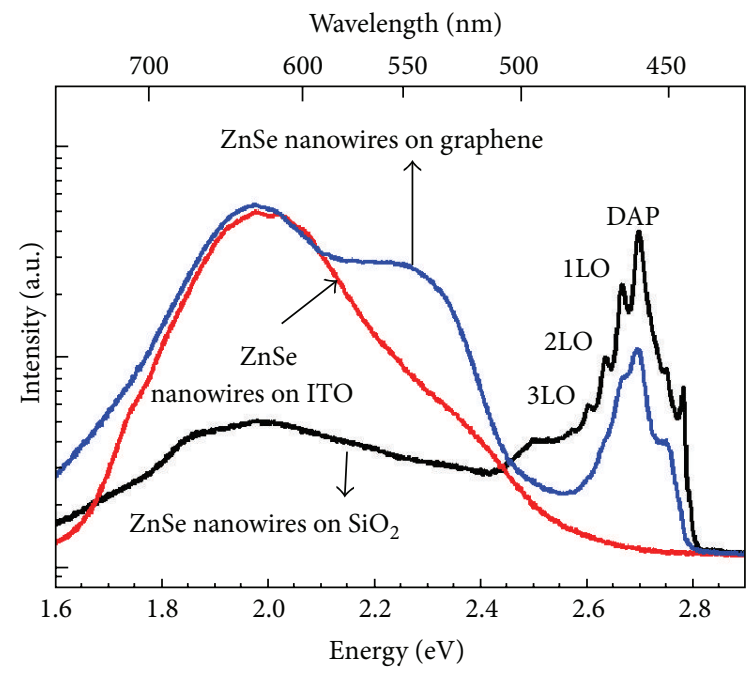

(a)

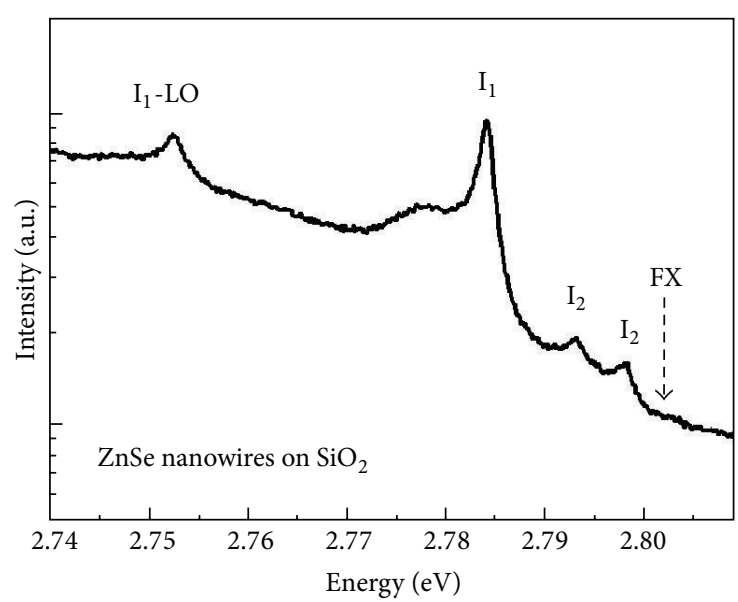

(b)

FIGURE 3: (a) PL spectra of ZnSe nanowires grown on $\mathrm{SiO}_{2}$, ITO, and graphene. (b) Expanded view of the high-energy side of the PL spectrum of $\mathrm{ZnSe}$ nanowires grown on $\mathrm{SiO}_{2}$.

plot. The $\beta$ value was $\sim 7,250$, which is higher than those previously reported for ZnSe nanoribbons ( 1382) [29], ZnO nanoneedles on plastic substrates ( 1134) [30], and carbon nanotubes on polyamide ( 850) [31]. These results indicate that ZnSe FEDs with graphene electrodes could be used to fabricate novel FE devices. The inset of Figure 4(a) illustrates a schematic of a FED structure employing $\mathrm{ZnSe}$ nanowires grown on graphene. Carbon nanotube (CNT)/ITO and $\mathrm{ZnSe}$ nanowire/graphene materials were used as the emitter/ cathode and phosphor/anode, respectively. Note that the sheet resistance of the graphene electrode was $\sim 2.0 \mathrm{k} \Omega / \mathrm{sq}$.
The field-emission properties of the device were measured in a diode configuration, using a $500 \mu \mathrm{m}$ gap between the anode and cathode. During measurements, the pressure was maintained at $\sim 1.5 \times 10^{-6}$ Torr.

Figure 4(b) shows the brightness of $\mathrm{ZnSe}$ nanowire/ graphene as a function of emission current. Figure 4(b) shows that the brightness of the $\mathrm{ZnSe}$ nanowire phosphor emissions increased from 20 to $80 \mathrm{~cd} / \mathrm{m}^{2}$ when the emission current was increased from 20 to $100 \mu \mathrm{A}$. The luminous efficiency was $0.87 \mathrm{~lm} / \mathrm{W}$ at $2.2 \mathrm{kV} / \mathrm{mm}$, higher than that reported for $\mathrm{SrTiO}_{3}: \mathrm{Pr}^{3+}(0.4 \mathrm{~lm} / \mathrm{W})$ [32]. The insets in Figure 4(b) show 


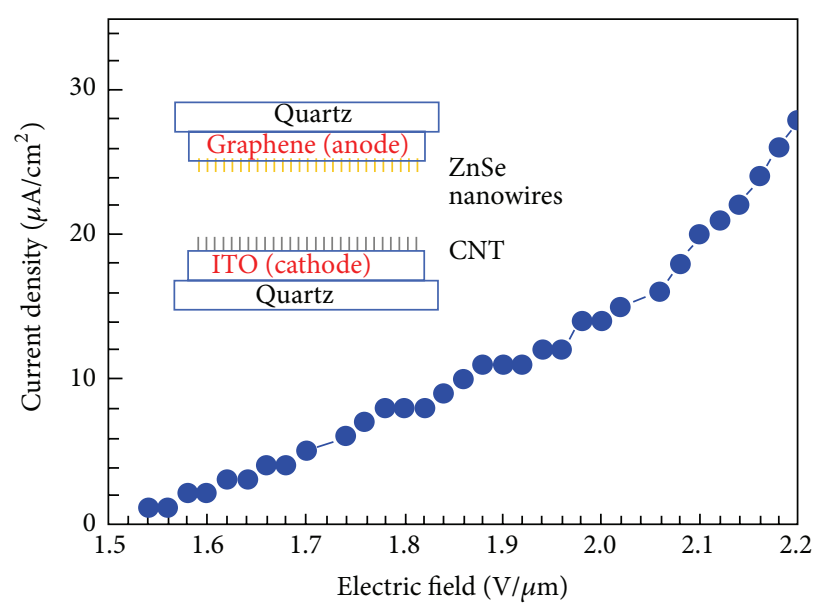

(a)

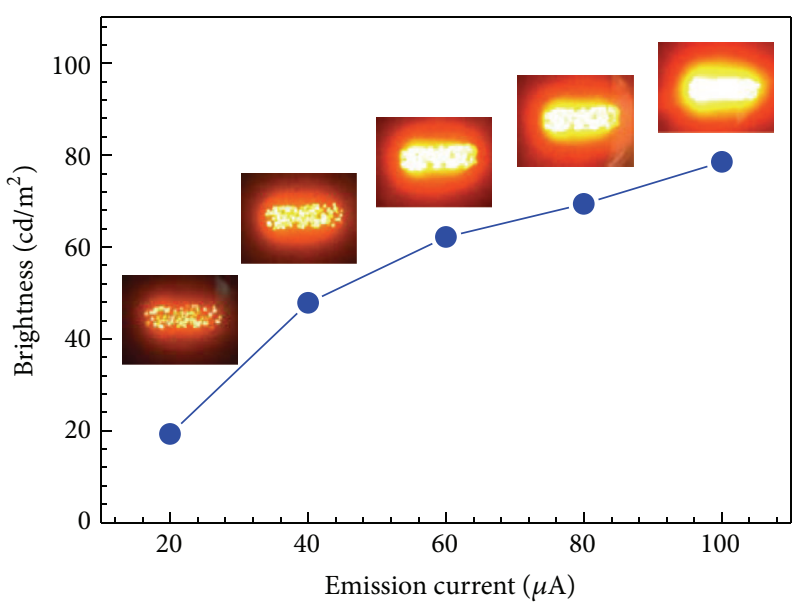

(b)

FIGURE 4: (a) Field-emission current versus applied electric field for a FED device constructed of ZnSe nanowires on graphene. The inset shows a schematic of the field-emitting structure used, which employs CNT/ITO and ZnSe nanowire/graphene as its emitter/cathode and phosphor/anode, respectively. (b) Light-emission luminance graph of a ZnSe nanowire phosphor on graphene. Insets are optical images of the red light emissions of the ZnSe/graphene device. Peak emission occurred at $\sim 627 \mathrm{~nm}$.

images of the light emissions of the device at each applied emission current, the size of the area imaged being $\sim 10 \times$ $20 \mathrm{~mm}$. The emission spectrum of the $\mathrm{ZnSe}$ nanowires shows an emission peak at $\sim 627 \mathrm{~nm}$. The average corresponding Commission Internationale de L'Eclairage (CIE) coordinates for the device are $(0.621,0.315)$.

\section{Conclusions}

In summary, $\mathrm{ZnSe}$ nanowires of varying crystallinity and luminescent properties were synthesized on three different substrates, $\mathrm{SiO}_{2}$, ITO, and graphene. XRD and HR-TEM analyses revealed that the $\mathrm{ZnSe}$ nanowires grown on $\mathrm{SiO}_{2}$ and graphene by CVD, using Au catalyst, had single-phase, cubic structures. On the other hand, the ZnSe nanowires grown on ITO showed the presence of both cubic and hexagonal structures. Furthermore, PL results for $\mathrm{ZnSe}$ nanowires on $\mathrm{SiO}_{2}$ and ITO exhibited remarkably high peaks at $2.701 \mathrm{eV}$ $(459 \mathrm{~nm})$ and $1.977 \mathrm{eV}(627 \mathrm{~nm})$, respectively, whereas those of $\mathrm{ZnSe}$ nanowires on graphene showed red luminescence at approximately $1.977 \mathrm{eV}(627 \mathrm{~nm})$ and a blue peak at $2.695 \mathrm{eV}$ $(460 \mathrm{~nm})$. A FED device was fabricated with light-emitting $\mathrm{ZnSe}$ nanowires on graphene. The fabricated FED device showed a red emission peak at $\sim 627 \mathrm{~nm}$ with CIE coordinates of $(0.621,0.315)$. Based on these experimental results, it is highly probable that a hybrid device made from nanowires and graphene could provide an effective means for developing highly functional and efficient light-emitting displays.

\section{Conflict of Interests}

The authors declare that there is no conflict of interests regarding the publication of this paper.

\section{Acknowledgments}

This work was supported by the National Research Foundation of Korea (NRF) grant funded by the Korea government (MSIP) (no. 2014R1A2A1A11049450). This work at Dankook University was supported by Leading Foreign Research Institute Recruitment Program through the National Research Foundation of Korea (NRF) funded by the Ministry of Education, Science and Technology (MEST) (no. 2014-039452).

\section{References}

[1] X. Duan, Y. Huang, R. Agarwal, and C. M. Lieber, "Singlenanowire electrically driven lasers," Nature, vol. 421, no. 6920, pp. 241-245, 2003.

[2] B. Tian, X. Zheng, T. J. Kempa et al., "Coaxial silicon nanowires as solar cells and nanoelectronic power sources," Nature, vol. 449, no. 7164, pp. 885-889, 2007.

[3] S. Ju, A. Facchetti, Y. Xuan et al., "Fabrication of fully transparent nanowire transistors for transparent and flexible electronics," Nature Nanotechnology, vol. 2, no. 7, pp. 378-384, 2007.

[4] S. Ju, J. Li, J. Liu et al., "Transparent active matrix organic lightemitting diode displays driven by nanowire transistor circuitry," Nano Letters, vol. 8, no. 4, pp. 997-1004, 2008.

[5] R. Könenkamp, R. C. Word, and C. Schlegel, "Vertical nanowire light-emitting diode," Applied Physics Letters, vol. 85, no. 24, pp. 6004-6006, 2004.

[6] M.-C. Jeong, B.-Y. Oh, M.-H. Ham, and J.-M. Myoung, "Electroluminescence from $\mathrm{ZnO}$ nanowires in $n-\mathrm{ZnO}$ film/ZnO nanowire array/p-GaN film heterojunction lightemitting diodes," Applied Physics Letters, vol. 88, no. 20, Article ID 202105, 2006.

[7] K. S. Kim, Y. Zhao, H. Jang et al., "Large-scale pattern growth of graphene films for stretchable transparent electrodes," Nature, vol. 457, no. 7230, pp. 706-710, 2009. 
[8] X. Wang, L. Zhi, and K. Müllen, "Transparent, conductive graphene electrodes for dye-sensitized solar cells," Nano Letters, vol. 8, no. 1, pp. 323-327, 2008.

[9] X. Li, Y. Zhu, W. Cai et al., "Transfer of large-area graphene films for high-performance transparent conductive electrodes," Nano Letters, vol. 9, no. 12, pp. 4359-4363, 2009.

[10] J. O. Hwang, J. S. Park, D. S. Choi et al., "Workfunction-tunable, $\mathrm{N}$-doped reduced graphene transparent electrodes for highperformance polymer light-emitting diodes," ACS Nano, vol. 6, no. 1, pp. 159-167, 2012.

[11] F. Bonaccorso, Z. Sun, T. Hasan, and A. C. Ferrari, "Graphene photonics and optoelectronics," Nature Photonics, vol. 4, no. 9, pp. 611-622, 2010.

[12] D. Choi, M.-Y. Choi, W. M. Choi et al., "Fully rollable transparent nanogenerators based on graphene electrodes," Advanced Materials, vol. 22, no. 19, pp. 2187-2192, 2010.

[13] L. Wang, M. Lu, X. Wang et al., “Tuning the p-type conductivity of $\mathrm{ZnSe}$ nanowires via silver doping for rectifying and photovoltaic device applications," Journal of Materials Chemistry A, vol. 1, no. 4, pp. 1148-1154, 2013.

[14] S. B. Mirov, V. V. Fedorov, K. Graham, I. S. Moskalev, V. V. Badikov, and V. Panyutin, "Erbium fiber laser-pumped continuous-wave microchip $\mathrm{Cr}^{2+}: \mathrm{ZnS}$ and $\mathrm{Cr}^{2+}: \mathrm{ZnSe}$ lasers," Optics Letters, vol. 27, no. 11, pp. 909-911, 2002.

[15] Y.-C. Zhu and Y. Bando, "Preparation and photoluminescence of single-crystal zinc selenide nanowires," Chemical Physics Letters, vol. 377, no. 3-4, pp. 367-370, 2003.

[16] P. K. Mohseni, A. Behnam, J. D. Wood et al., " $\operatorname{In}_{\mathrm{x}} \mathrm{Ga}_{1-\mathrm{x}}$ As nanowire growth on graphene: Van der waals epitaxy induced phase segregation," Nano Letters, vol. 13, no. 3, pp. 1153-1161, 2013.

[17] J. P. Alper, A. Gutes, C. Carraro, and R. Maboudian, "Semiconductor nanowires directly grown on graphene-towards wafer scale transferable nanowire arrays with improved electrical contact," Nanoscale, vol. 5, no. 10, pp. 4114-4118, 2013.

[18] J. Bong, J. Han, J. Lee, S. Kim, and S. Ju, "Fabrication of highly transparent nanowire transistors with one-stepprocessed graphene gate-source-drain electrodes," Applied Physics Express, vol. 6, no. 5, Article ID 055103, 2013.

[19] N. Shibata, A. Ohki, S. Zembutsu, and A. Katsui, "Photoluminescence due to lattice-mismatch defects in high-purity $\mathrm{ZnSe}$ layers grown by metalorganic vapor phase epitaxy," Japanese Journal of Applied Physics, Part 2: Letters, vol. 27, no. 3, pp. 441443, 1988.

[20] K. Shahzad, "Excitonic transitions in ZnSe epilayers grown on GaAs," Physical Review B, vol. 38, no. 12, pp. 8309-8312, 1988.

[21] D. G. Thomas and J. J. Hopfield, "Optical properties of bound exciton complexes in cadmium sulfide," Physical Review, vol. 128, no. 5, pp. 2135-2148, 1962.

[22] C. Ye, X. Fang, Y. Wang, P. Yan, J. Zhao, and L. Zhang, "Structural characterization of long ZnSe nanowires," Applied Physics A, vol. 79, no. 1, pp. 113-115, 2004.

[23] C. H. Hsiao, S. J. Chang, S. B. Wang et al., "ZnSe nanowire photodetector prepared on oxidized silicon substrate by molecularbeam epitaxy," Journal of the Electrochemical Society, vol. 156, no. 4, pp. J73-J76, 2009.

[24] Y. Jiang, X.-M. Meng, W.-C. Yiu et al., "Zinc selenide nanoribbons and nanowires," The Journal of Physical Chemistry B, vol. 108, no. 9, pp. 2784-2787, 2004.

[25] Q. Li, X. Gong, C. Wang, J. Wang, K. Ip, and S. Hark, "Sizedependent periodically twinned ZnSe nanowires," Advanced Materials, vol. 16, no. 16, pp. 1388-1440, 2004.
[26] Y. Q. Wang, U. Philipose, T. Xu, H. E. Ruda, and K. L. Kavanagh, "Twinning modulation in ZnSe nanowires," Semiconductor Science and Technology, vol. 22, no. 3, pp. 175-178, 2007.

[27] R. K. Swank, "Surface properties of II-VI compounds," Physical Review, vol. 153, no. 3, pp. 844-849, 1967.

[28] Z.-G. Chen, L. Cheng, H.-Y. Xu et al., "ZnS branched architectures as optoelectronic devices and field emitters," Advanced Materials, vol. 22, no. 21, pp. 2376-2380, 2010.

[29] L. Zhao, Q. Pang, Y. Cai et al., "Vertically aligned zinc selenide nanoribbon arrays: microstructure and field emission," Journal of Physics D: Applied Physics, vol. 40, no. 12, pp. 3587-3591, 2007.

[30] H. Y. Yang, S. P. Lau, S. F. Yu et al., "Field emission from zinc oxide nanoneedles on plastic substrates," Nanotechnology, vol. 16, no. 8, pp. 1300-1303, 2005.

[31] S. Hofmann, C. Ducati, B. Kleinsorge, and J. Robertson, "Direct growth of aligned carbon nanotube field emitter arrays onto plastic substrates," Applied Physics Letters, vol. 83, no. 22, pp. 4661-4663, 2003.

[32] H. Yamamoto and S. Okamoto, "Efficiency enhancement by aluminum addition to some oxide phosphors for field emission displays," Displays, vol. 21, no. 2-3, pp. 93-98, 2000. 

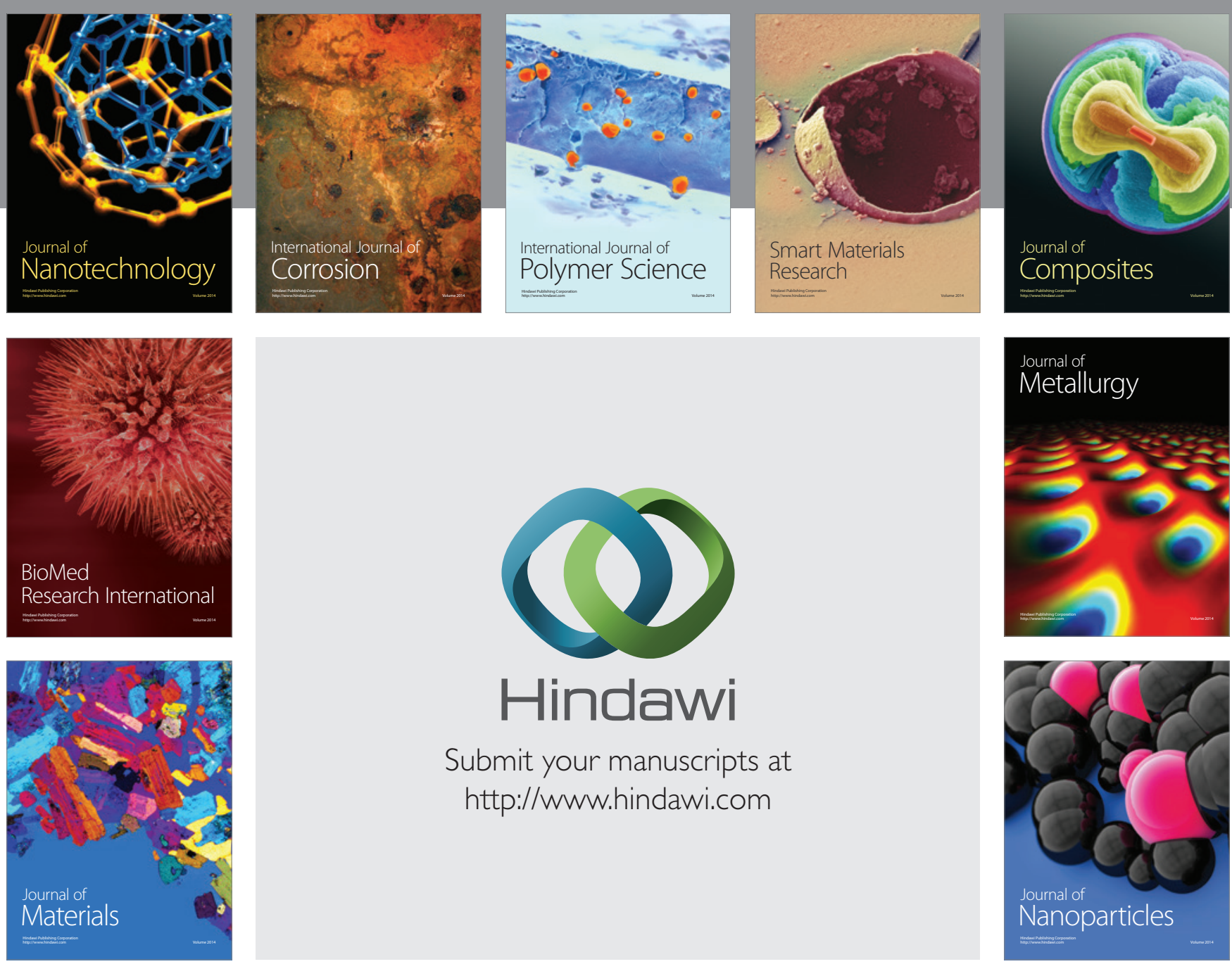

Submit your manuscripts at http://www.hindawi.com
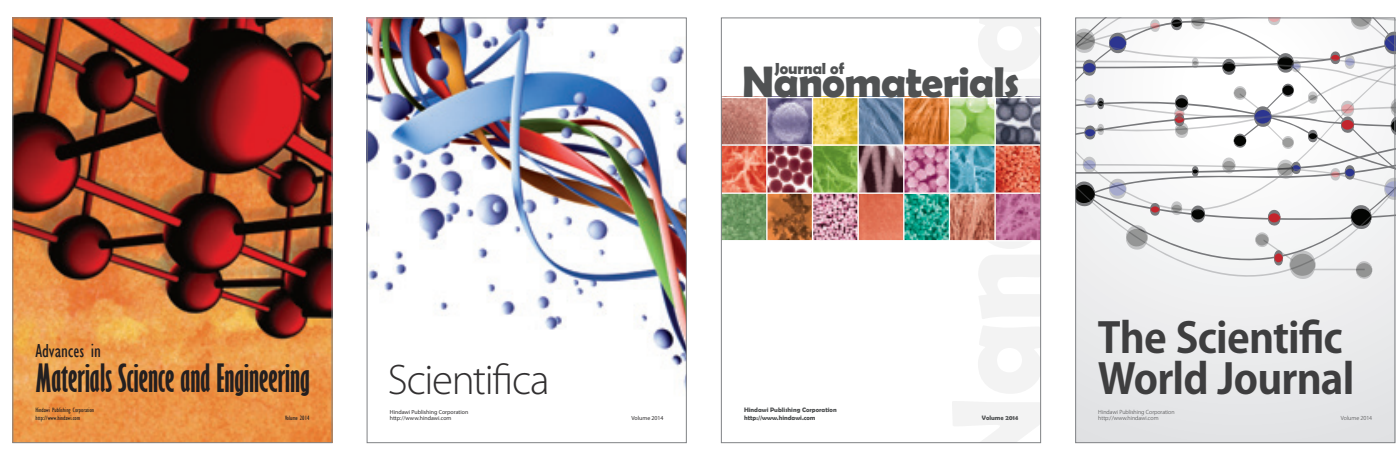

\section{The Scientific World Journal}
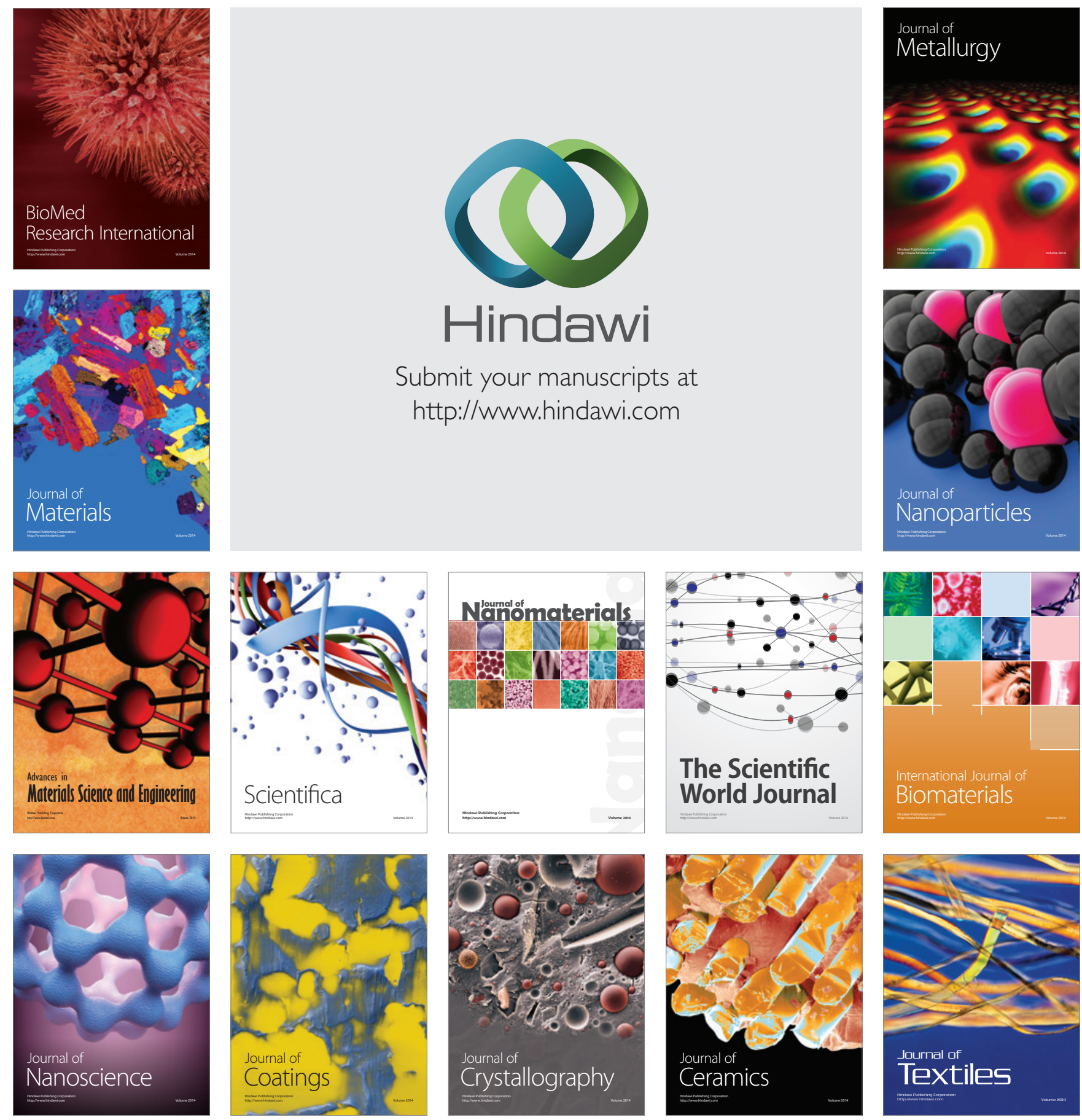